\title{
Autochthonous Thelazia callipaeda Infection in Dog, New York, USA, 2020
}

\author{
A.B. Schwartz, ${ }^{1}$ Manigandan Lejeune, ${ }^{1}$ Guilherme G. Verocai, Rebecca Young, Paul H. Schwartz
}

We report a case of autochthonous infection of the eye worm Thelazia callipaeda in a dog in the northeastern United States. Integrated morphologic identification and molecular diagnosis confirmed the species. Phylogenetic analysis suggested introduction from Europe. The zoonotic potential of this parasite warrants broader surveillance and increased awareness among physicians and veterinarians.

$\mathrm{T}$

Thelaziasis in dogs can be caused by 2 nematodes of 1 the genus Thelazia (Nematoda: Spirurida): T. callipaeda and T. californiensis (1). The oriental eye worm (T. callipaeda) is a helminth that infects a variety of domestic and wild carnivores, lagomorphs, rodents, and primates (including humans) across Eurasia $(2,3)$. In Europe, the T. callipaeda eye worm is an emergent vectorborne helminth that has spread steadily across all countries over the past 3 decades (2). The California eye worm (T. californiensis) has been reportedly found in wild and domestic carnivores, ungulates, lagomorphs, and humans; its range is limited to the western United States (4). Zoonotic infection of humans with a third species of eye worm (T. gulosa), which infects cattle, has recently been reported in the western United States (5). These 3 species of Thelazia eye worm with zoonotic potential are morphologically and biologically distinct $(1,5,6)$.

Thelazia nematodes are found in the conjunctival recesses of the eye (1). Secretophagous dipteran intermediate hosts $(1,7)$ ingest first-stage larvae (L1) while feeding from the definitive host's eyes. After

Author affiliations: Marist College, Poughkeepsie, New York, USA (A.B. Schwartz); Animal College of Veterinary Medicine at Cornell University, Ithaca, New York, USA (M. Lejeune, R. Young); Texas A\&M University College of Veterinary Medicine and Biological Sciences, College Station, Texas, USA (G.G. Verocai); Center for Veterinary Care Millbrook, PC, Millbrook, New York, USA (P.H. Schwartz)

DOI: https://doi.org/10.3201/eid2707.210019 metamorphosis, infective third-stage larvae (L3) are passed via the labelum onto the conjunctiva of another suitable host. L3 develop into adults that migrate to the conjunctival recess, lacrimal ducts, or both, resulting in conjunctivitis, ocular discharge, and blepharospasm. Female worms release more L1, seeding ocular secretions of the host, and conclude the life cycle $(1,2)$. Intermediate hosts for Thelazia nematodes are dipteran flies of the genera Phortica for T. callipaeda, Fannia for T. californiensis, and Musca for T. gulosa $(1,5,7,8)$. P. variegata fruit flies are widely distributed across Eurasia and have been found in multiple areas in the eastern United States (9). In North America, they have been experimentally proven to be competent vectors for T. callipaeda worms (10), supporting the potential occurrence of $T$. callipaeda infection in the United States (2). We report T. callipaeda eye worm infection detected in a dog in the Western Hemisphere in November 2020.

\section{The Case}

The patient was a 7.5-year-old Labrador retriever, with no relevant medical history. The dog routinely received heartworm preventive (Heartgard; Boehringer Ingelheim Pharmaceuticals, Inc., https:// www.boehringer-ingelheim.com) and flea and tick preventive (NexGard [Boehringer Ingelheim Pharmaceuticals, Inc.] and Vectra [Ceva Animal Health, https://www.ceva.us]) in accordance with recommended dosing and had never traveled beyond Dutchess County, New York, USA. The dog was taken to a veterinarian because of a 3-week history of unilateral epiphora and blepharospasm. Treatment with an ophthalmic preparation (neomycin, polymyxin B, and dexamethasone) produced no demonstrable response. Subsequent nasolacrimal duct flushing with $0.3 \%$ gentamicin sulfate and $0.2 \%$ dexamethasone in physiological saline solution, followed by $100 \mu \mathrm{g} /$

\footnotetext{
${ }^{1}$ These authors contributed equally to this article.
} 
$\mathrm{mL}$ ivermectin in physiological saline solution, led to recovery of 12 nematodes. After systemic ivermectin administration, no recurrence has been noted.

Four nematodes ( 3 female, 1 male) were morphologically identified as T. callipaeda eye worms on the basis of the cuticular transverse striations (CTS) pattern and vulva position $(1,6)$. The 3 female worms were 12.7-13.9 $\mathrm{mm}$ long and 314-360 $\mu \mathrm{m}$ wide. The vulval opening was anterior to the esophageal intestinal junction, and in 1 specimen it was $610.86 \mu \mathrm{m}$ from the cephalic end. The midbodies contained 150-190 $\mathrm{CTS} / \mathrm{mm}$, and the cephalic/caudal region contained 220-240 CTS/mm. The buccal capsule was wider than it was deep. Two protruding phasmids were visible at the tip of the tail, which did not taper unilaterally (Figure 1). The male worm was $8.9 \mathrm{~mm}$ long, and its width was not measured. The cephalic region contained $310 \mathrm{CTS} / \mathrm{mm}$, and the midbody/caudal region contained $170 \mathrm{CTS} / \mathrm{mm}$. The small spicules measured $147.73 \mu \mathrm{m}$; the large spicules, $1721.90 \mu \mathrm{m}$.

We subjected a female worm to DNA extraction and multilocus PCR (18S rRNA, $12 \mathrm{~S}$ rRNA, and cytochrome oxidase c subunit 1 [ $\operatorname{cox} 1$ ] gene markers) by using assays described previously $(4,11)$, followed by sequencing. The partial sequences generated for $18 \mathrm{~S}$ rRNA matched $99.7 \%$, $12 \mathrm{~S}$ rRNA $99.4 \%$, and cox 1 genes $93.3 \%-100 \%$ of the corresponding genes of $T$. callipaeda worms in GenBank. We deposited the generated sequences in GenBank (accession no. MW570771 for 18S rRNA, MW575766 for 12S rRNA, and MW570733 for cox1). Molecular data unequivocally confirmed the parasite as T. callipaeda. Phylogenetic analysis of the cox 1 gene showed that the T. callipaeda eye worm found in North America belongs to the haplotype- 1 prevalent in Europe $(100 \%$ maximum identity), suggesting a possible source of introduction (Figure 2).

\section{Conclusions}

The discovery of an autochthonous case of T. callipaeda eye worm infection in the United States suggests its introduction and establishment on a continent where natural infection has not been documented. The report on the distribution of $P$. variegata fruit flies in the eastern United States and their competence for T. callipaeda eye worms has raised concern for eye worm infections in animals and humans in this region (10). Our finding is in line with previous predictions. Active surveillance of all susceptible hosts, coupled with ecologic niche modeling as conducted in Europe, can help gauge the extent of T. callipaeda eye worm spread in North America (7). Our findings should bring awareness about this invasive, zoonotic parasite to veterinary and medical ophthalmologists in the Americas. To curtail the potential spread in the United States, consideration should be given to US Department of Agriculture-imposed

Figure 1. Integrated diagnostic approach for confirming Thelazia callipaeda nematodes: morphologic identification. Specimens were cleared in lactophenol before examination under an Olympus compound microscope (BX53) (https:// www.olympus-lifescience.com). Images were taken with an Olympus DP73 camera, and morphometry was performed by using Olympus cellSens software. A) Cephalic end of a female worm. Black arrow indicates esophageal intestinal junction; red arrow indicates vulval opening. Original magnification $\times 100$. B) Transverse striations (150-190/ $\mathrm{mm}$ ) in the cuticle of midbody region of a female worm. Original magnification $\times 200$. C) Buccal cavity of a female worm, wider than deep. Note tightly spaced cuticular striations in the cephalic end. Original magnification $\times 200$. D) Caudal

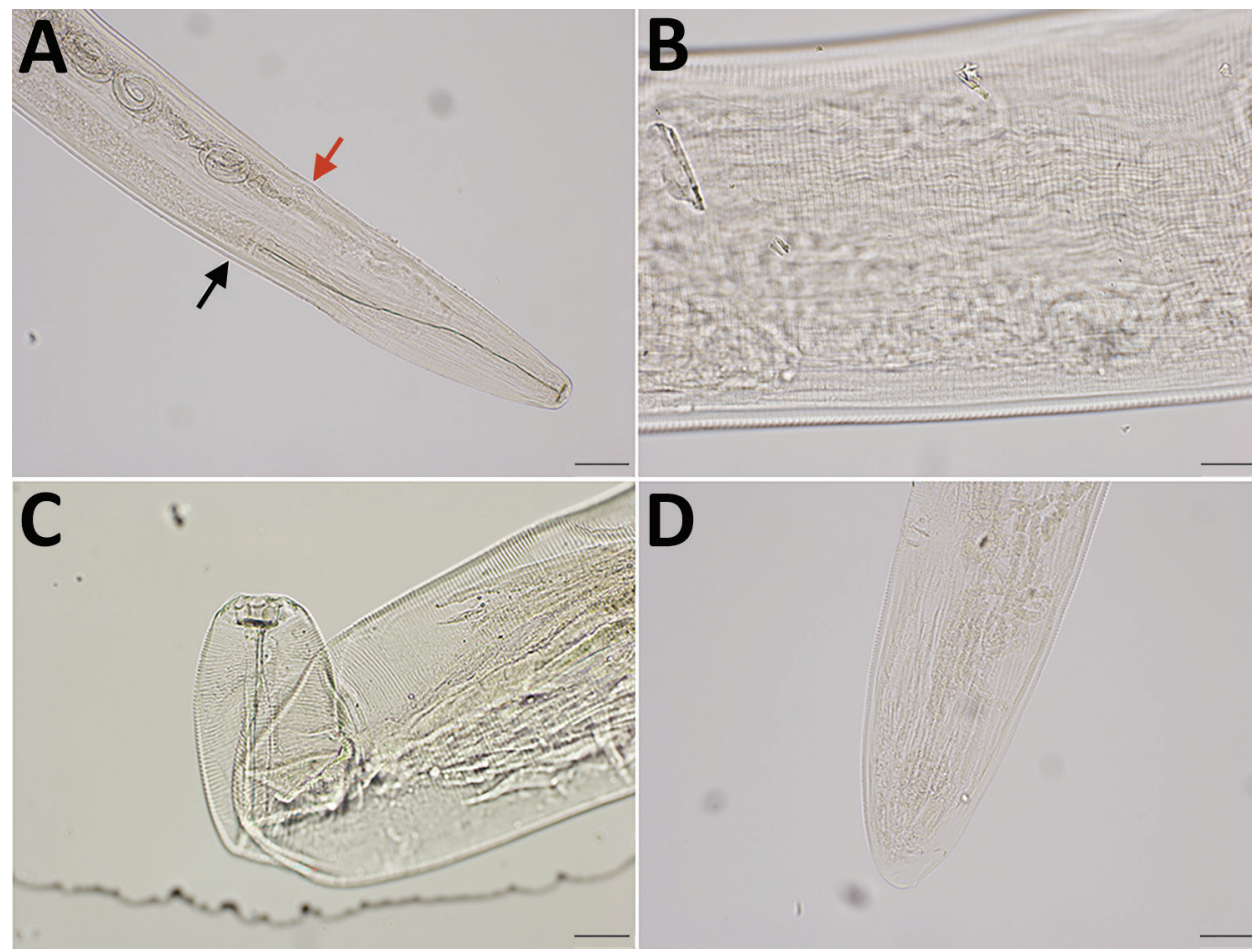
end of female worm with protruding phasmids in the tip. The tail was not protruding unilaterally. Original magnification $\times 100$. 


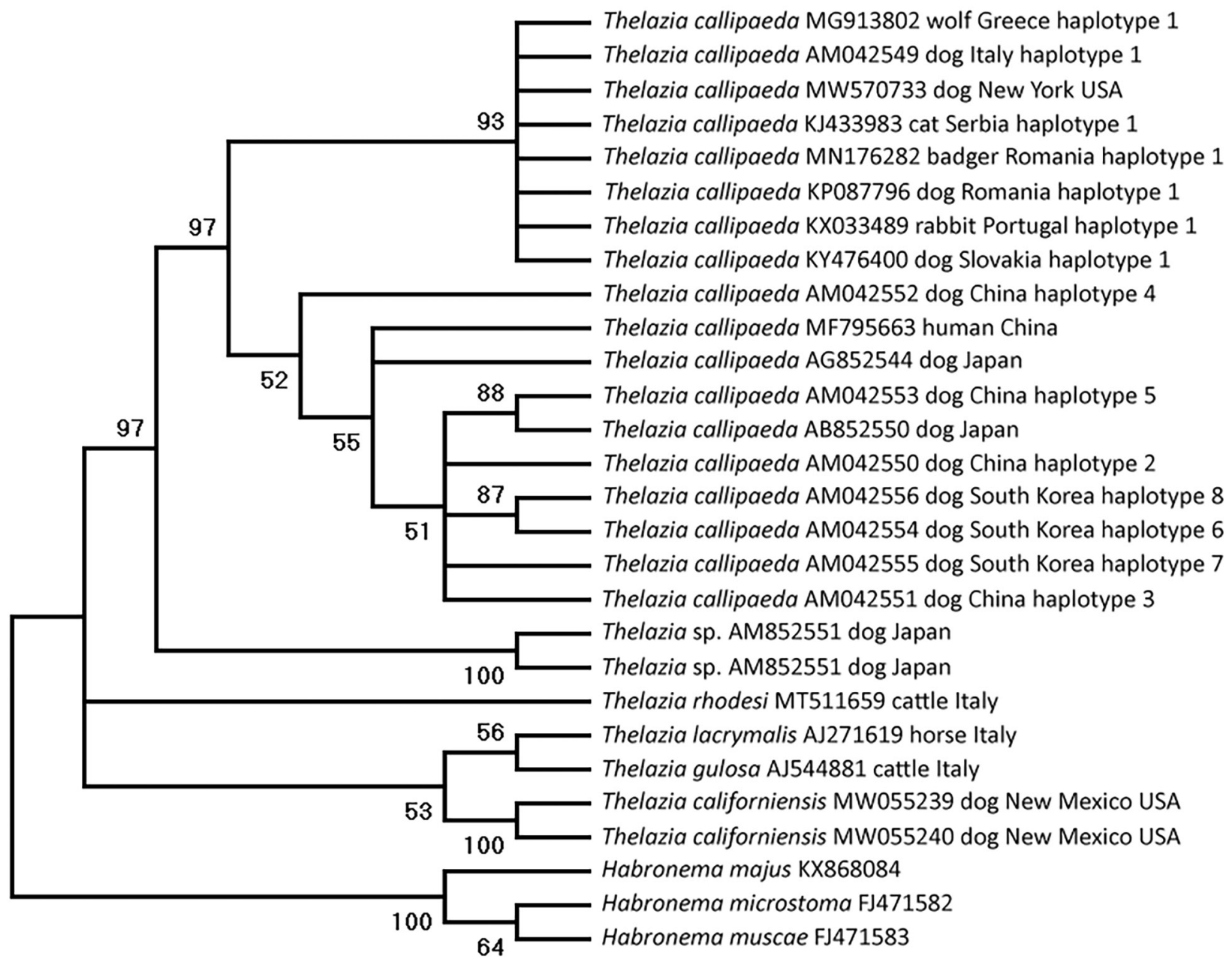

Figure 2. Phylogenetic relationship based on cytochrome oxidase c subunit 1 gene of Thalazia callipaeda nematode isolate from a dog in Dutchess County, New York, USA, 2020 (GenBank accession no. MW570733), and other related Thelazia species available in GenBank (accession numbers shown). Analysis was performed by using MEGAX (http://www.megasoftware.net) and the maximumlikelihood method, 1,000 bootstrap replicates; nodes with $<50 \%$ support were condensed.

requirements for implementing broad and accurate parasite diagnostic methods and prophylactic anthelmintic treatment to mitigate the introduction of exotic parasites before relevant species are imported. The One Health model of cooperation among veterinarians, wildlife biologists, and physicians is vital for assessing the current distribution and mitigating the spread of this multihost parasite with zoonotic potential. As this case shows, emergence of T. callipaeda parasites requires increased awareness by both human medical and veterinary professionals.

\section{Acknowledgments}

We thank Caroline Sobotyk de Oliveira for her assistance depositing DNA sequences in GenBank, Domenico Otranto for his assistance with elucidating the potential extension of this emergent disease, and Antech
Diagnostics for sharing information about cases of Thelazia infection identified in their laboratory.

\section{About the Author}

Ms. A.B. Schwartz is a graduate of Marist College with an interest in biopharmaceutical development; she will begin a master's degree in Public Health at Georgetown University in the fall of 2021.

\section{References}

1. Naem S. Thelazia species and conjunctivitis. In: Z. Pelikan, editor. Conjunctivitis - A Complex and Multifaceted Disorder. Rijeka (Croatia): Intech Open Access Press; 2011. p. 201-32.

2. Otranto D, Mendoza-Roldan JA, Dantas-Torres F. Thelazia callipaeda. Trends Parasitol. 2021;37:263-4. https:/ / doi.org/ 10.1016/j.pt.2020.04.013 
3. Otranto D, Traversa D. Thelazia eyeworm: an original endo- and ecto-parasitic nematode. Trends Parasitol. 2005;21:1-4. https://doi.org/10.1016/j.pt.2004.10.008

4. Sobotyk C, Foster T, Callahan RT, McLean NJ, Verocai GG. Zoonotic Thelazia californiensis in dogs from New Mexico, USA, and a review of North American cases in animals and humans. Vet Parasitol Reg Stud Rep. 2021;24:100553. https://doi.org/10.1016/j.vprsr.2021.100553

5. Bradbury RS, Gustafson DT, Sapp SGH, Fox M, de Almeida M, Boyce $\mathrm{M}$, et al. A second case of human conjunctival infestation with Thelazia gulosa and a review of T. gulosa in North America. Clin Infect Dis. 2020;70:518-20.

6. Kofoid CA, Williams OL. The nematode Thelazia californiensis as a parasite of the eye of man in California. Arch Ophthalmol. 1935;13:176-80. https://doi.org/10.1001/ archopht.1935.00840020036002

7. Otranto D, Brianti E, Cantacessi C, Lia RP, Máca J. The zoophilic fruitfly Phortica variegata: morphology, ecology and biological niche. Med Vet Entomol. 2006;20:358-64. https://doi.org/10.1111/j.1365-2915.2006.00643.x

8. Otranto D, Cantacessi C, Testini G, Lia RP. Phortica variegata as an intermediate host of Thelazia callipaeda under natural conditions: evidence for pathogen transmission by a male arthropod vector. Int J Parasitol. 2006;36:1167-73. https://doi.org/10.1016/j.ijpara.2006.06.006

9. Werner T, Jaenike J. The encyclopedia of north american drosophilids: drosophilids of the midwest and northeast, 2017 [cited 2021 May 10]. https://digitalcommons.mtu.edu/ cgi/viewcontent.cgi?article $=1000 \&$ context $=$ oabooks

10. Otranto D, Iatta R, Lia RP, Cavalera MA, Màca J, Pombi M, et al. Competence of Phortica variegata from the United States as an intermediate host of the Thelazia callipaeda eyeworm. Am J Trop Med Hyg. 2018;98:1175-8. https://doi.org/10.4269/ajtmh.17-0956

11. Floyd RM, Rogers AD, Lambshead PJD, Smith CR. Nematode specific PCR primers for the $18 \mathrm{~S}$ small subunit rRNA gene. Mol Ecol Notes. 2005;5:611-2. https:/ / doi.org/ 10.1111/j.1471-8286.2005.01009.x

Address for correspondence: Paul H. Schwartz and A.B. Schwartz, Center for Veterinary Care Millbrook, PC, PO Box 518, Millbrook, NY 122545, USA: email: cvcroute343@gmail.com and alex.barnes. schwartz@gmail.com or hvsevc@gmail.com

\section{May 2021 COVID-19}

- Coordinated Strategy for a Modeling-Based Decision Support Tool for COVID-19, Utah, USA

- Clinical Laboratory Perspective on Human Infections Caused by Unusual Nonhemolytic, Lancefield Group B Streptococcus halichoeri

- Case Series of LaboratoryAssociated Zika Virus Disease, United States, 2016-2019

- Successful Control of an Onboard COVID-19 outbreak Using the Cruise Ship as a Quarantine Facility, Western Australia T

- Coccidioidomycosis and COVID-19 Co-Infection, United States, 2020

- Epidemiologic Findings From Case Investigations and Contact Tracing of the First 200 Cases of Coronavirus Disease 2019 (COVID-19) identified in Santa Clara County, California, USA

- SARS-CoV-2 in Nursing Homes after 3 Months of Serial, Facility-Wide Point Prevalence Testing, Connecticut, USA

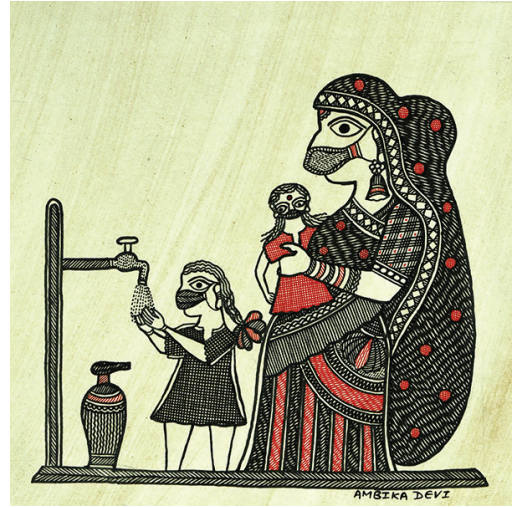

- Transmission of Severe Acute Respiratory Syndrome Coronavirus 2 during Border Quarantine and Air Travel, New Zealand (Aotearoa)

- Herd Immunity against Severe Acute Respiratory Syndrome Coronavirus 2 Infection in 10 Communities, Qatar

- Characteristics and Clinical Implications of CarbapenemaseProducing Klebsiella pneumoniae Colonization and Infection, Italy
- Serologic Screening of Severe Acute Respiratory Syndrome Coronavirus 2 Infection in Cats and Dogs during First Coronavirus Disease Wave, the Netherlands

- Epidemiologic History and Genetic Diversity Origins of Chikungunya and Dengue Viruses, Paraguay

- Monitoring SARS-CoV-2 Circulation and Diversity through Community Wastewater Sequencing, the Netherlands and Belgium

- Active Case Finding of Current Bornavirus Infections in Human Encephalitis Cases of Unknown Etiology, Germany, 2018-2020

- Susceptibility to SARS-CoV-2 of Cell Lines and Substrates Commonly Used to Diagnose and Isolate Influenza and Other Viruses

- Symptom Diary-Based Analysis of COVID-19 Disease Course, Germany, 2020

- Use of Genomics to Track Coronavirus Disease Outbreaks, New Zealand 